\title{
Relationship between sampling and multirate filterbanks in the linear canonical transform domain
}

\author{
Feng Zhang, Ran Tao ${ }^{*}$ and Yue Wang
}

\begin{abstract}
Multirate filterbanks have found applications in speech processing, image processing, communications, and in the development of new sampling theorems. This paper explores the relationship between sampling theorems and multirate filterbanks in the linear canonical transform (LCT) domain. The sampling identity and the interpolation identity for bandlimited signals in the LCT domain are discussed and then employed to obtain a discrete-time implementation for bandlimited signals in the LCT domain from their multichannel samples. Furthermore, we relate the multichannel sampling for bandlimited signals in the LCT domain as well as the corresponding continuous-time LCT filterbank to the discrete-time LCT filterbank, which can lead to new sampling strategies for bandlimited signals in the LCT domain.
\end{abstract}

Keywords: Linear canonical transform, Time-frequency analysis, Sampling theorem, Filterbank, Sampling rate conversion

\section{Introduction}

Over the recent years, multirate filterbanks have found applications in speech processing, image processing, communications, radar systems, etc. $[1,2]$. The structure of uniform filterbanks is generally composed of two parts: an analysis and a synthesis filterbank. Filterbanks help split a signal into two or more subbands, decimate each subband signal, and allocate bits for samples in each subband, which is the basic principle of subband coding for speech and image signals [1]. Specifically, properties of speech and image signals to human perception are used in the coding process. In video signal processing, subband coding has been successfully applied for image compression [3-5].

Sampling is one of the most fundamental concepts in the area of digital signal processing, which serves as a bridge between continuous physical signals and discrete signals [6]. Sampling theorems for continuous-time signals remain an active research field for decades. The classical Shannon sampling theorem has been extended into various generalized versions. Specifically, Papoulis has showed

\footnotetext{
* Correspondence: rantao@bit.edu.cn

Department of Electronic Engineering, Beijing Institute of Technology, Beijing 100081, China
}

that a Fourier bandlimited signal can be recovered from several linear time-invariant filtered versions of the original signal, where each channel is sampled at a fraction of the Nyquist rate [7]. In fact, the Papoulis' multichannel sampling theorem can be understood from the framework of filterbanks, which is independently developed in the discrete-time domain. In [8], Brown exhibits the resemblance between the Papoulis' multichannel sampling and the filterbanks and shows that the reconstruction process from multichannel samples is essentially a problem of designing a synthesis filterbank. In [9], Vaidyanathan and Liu further exploits the relationship between the multichannel sampling theorem and multirate filterbanks, and utilizes the framework of the filterbanks as a convenient tool for the derivation of the sampling theorems. In [10], the filterbank interpretation of multichannel sampling is presented more explicitly by using two identities, and the efficient reconstruction from multichannel samples is obtained.

In another advance in signal processing, the linear canonical transform (LCT) has been introduced and used in processing nonstationary signals [11]. The LCT is a linear integral transform with four parameters $(a, b, c$, and $d$ ). Although it is not very well-known, its special

\section{穴}


cases are widely applied in various fields under different names [12]. The well-known operations such as Fourier transform (FT), the fractional Fourier transform (FRFT) [11], the Fresnel transform [13], and the scaling operation are all special cases of the LCT. The LCT is also applied in filter design, signal synthesis, pattern recognition, timefrequency analysis, holographic three-dimensional television, and many others [14-17]. Understanding the LCT can help gain more insight into its special cases and carry the knowledge gained from one subject to others [12]. Moreover, a nonstationary signal (for example, the chirp signals) that is nonbandlimited in the FT domain can be bandlimited in a LCT domain. Therefore, many results in the FT domain have been extended to the LCT domain, such as uncertainty relations $[18,19]$, sampling theorems [20-26], sampling rate conversion [27], and multirate filterbanks [28].

This paper generalizes the relationship between the multichannel sampling theorem and multirate filterbanks from the FT case to the LCT case. In section 2, some preliminaries about the definition of the LCT, the uniform sampling theorem, and the convolution theorem for the LCT are introduced. The sampling and interpolation identities for bandlimited signals in the LCT domain are presented in section 3. In section 4, we use these identities to obtain an efficient reconstruction method for bandlimited signals in the LCT domain from multichannel samples. Moreover, in section 4, we discuss the relationship between a continuous-time LCT filterbank, which is an interpretation of the multichannel sampling, and a discrete-time LCT (DTLCT) filterbank. Finally, conclusions are made in section 5 . The derived results reveal a method for processing signals nonbandlimited in the FT domain but bandlimited in the LCT domain, and can be seen as a step towards generalizing the relationship between the multichannel sampling and filterbanks from the FT to other transforms that are special cases of the LCT.

\section{Preliminaries}

\subsection{Linear canonical transform}

The LCT of $x(t)$ with parameters $(a, b, c$, and $d)$, namely $X^{\mathbf{M}}(u)$, is defined as [11]

$$
\begin{aligned}
X^{\mathrm{M}}(u) & =L^{\mathrm{M}}[x](u) \\
& =\left\{\begin{array}{c}
\sqrt{\frac{1}{j 2 \pi b}} \cdot e^{j \frac{d}{2 b} u^{2}} \int_{-\infty}^{\infty} x(t) e^{j} \frac{a}{2 b} t^{2}-j \frac{1}{b} u t d t, \quad b \neq 0 \\
\sqrt{d e} \frac{c d}{2} u^{2} x(d u), \quad b=0
\end{array},\right.
\end{aligned}
$$

where $\mathbf{M}=\left[\begin{array}{ll}a & b \\ c & d\end{array}\right]$ is the parameter matrix and $\operatorname{det}(\mathrm{M})=$ $\mathrm{ad}-\mathrm{bc}=1$. Two successive LCTs with matrices $\mathbf{M}_{1}$ and
$\mathbf{M}_{2}$ equal to another LCT with the matrix $\mathbf{M}_{3}=\mathbf{M}_{2} \mathbf{M}_{1}$; consequently, the inverse LCT is given by the LCT with parameters $(\mathrm{d},-\mathrm{b},-\mathrm{c}$, and $\mathrm{a})$. The LCT with the parameter matrix $\mathbf{M}=\left[\begin{array}{cc}\cos \theta & \sin \theta \\ -\sin \theta & \cos \theta\end{array}\right]$ reduces to the FRFT and to the FT when $\theta=\pi / 2$. The LCT reduces to the Fresnel transform when $\mathbf{M}=\left[\begin{array}{ll}1 & b \\ 0 & 1\end{array}\right]$. Note that when $\mathrm{b}=0$, the LCT becomes a chirp multiplication. Therefore, only the cases where $b \neq 0$ are considered. Without loss of generality, we assume $b>0$ in this paper.

A signal $x(t)$ is said to be bandlimited to $\sigma_{M}$ in the LCT domain with the parameter $M$ if $X^{\mathbf{M}}(\mathrm{u})=0$ for $|\mathrm{u}|>\sigma \mathrm{M}$. A nonzero bandlimited signal $\mathrm{x}(\mathrm{t})$ in the LCT domain has the following property: If $\mathrm{x}(\mathrm{t})$ is bandlimited in the LCT domain with the parameter $\mathbf{M}_{1}=\left[\begin{array}{ll}a_{1} & b_{1} \\ c_{1} & d_{1}\end{array}\right]$, then it cannot be bandlimited with another parameter $\mathbf{M}_{2}=\left[\begin{array}{ll}a_{2} & b_{2} \\ c_{2} & d_{2}\end{array}\right]$, satisfying the relationship $\mathrm{a} 1 / \mathrm{b} 1 \neq \mathrm{a} 2 /$ b2 [25]. From this property, we can see that if a signal is nonbandlimited in the FT domain, then it can be bandlimited in the LCT domain [29]. Therefore, the problems of processing nonbandlimited signals in the FT domain can be solved in the LCT domain.

\subsection{Uniform sampling theorem in the LCT domain}

The uniform sampled version of a continuous-time signal $x(t)$ with the sampling period $T$ is $x_{T}(t)=x(t)$ $\sum_{n=-\infty}^{\infty} \delta(t-n T)$, where $\delta(t)$ is the unit impulse function, or Dirac delta function. The spectrum in the LCT domain of the uniform sampled signal $x_{T}(t)$ is [16]

$$
\begin{aligned}
X_{T}^{\mathrm{M}}(u) & =L_{T}^{\mathrm{M}}[x](u)=L^{\mathrm{M}}\left[x(t) \sum_{n=-\infty}^{\infty} \delta(t-n T)\right] \\
& =\sqrt{\frac{1}{j 2 \pi b}} \cdot e^{j \frac{d}{2 b} u^{2}} \sum_{n=-\infty}^{\infty} x(n T) e^{j \frac{a}{2 b}(n T)^{2}-j \frac{1}{b} u(n T)} \\
& =\frac{1}{T} e^{j \frac{d}{2 b} u^{2}} \sum_{k=-\infty}^{\infty} X^{\mathrm{M}}\left(u-k \frac{2 \pi}{T} b\right) e^{-j \frac{d}{2 b}\left(u-k \frac{2 \pi}{T} b\right)^{2}} .
\end{aligned}
$$

Equation (2) shows that the LCT spectrum $X_{T}^{\mathrm{M}}(u)$ replicates $X^{\mathbf{M}}(\mathrm{u})$ with a period $2 \pi b / T$ along with a phase shift. Thus, the Nyquist sampling period for a bandlimited signal in the LCT domain with the parameter $\mathbf{M}$ is $T_{\mathrm{s}}^{\mathbf{M}}=\pi b / \sigma_{\mathbf{M}}$. For a discrete-time signal $x_{T}(n)$ sampled from a continuous-time signal $x(t)$ with the sampling period $T$, i.e., $x_{T}(n)=x(n T)$, the (DTLCT of $x_{T}(n)$ 
with parameters $(a, b, c$, and $d)$, namely $\tilde{X}_{T}^{\mathrm{M}}(w)$, can be defined from (2) by denoting $w=u T$ [27]:

$$
\begin{aligned}
\tilde{X}_{T}^{\mathbf{M}}(w) & =\tilde{L}_{T}^{\mathbf{M}}[x](w) \\
& =\sqrt{\frac{1}{j 2 \pi b}} \cdot e^{j \frac{d}{2 b T^{2}}} \sum_{n=-\infty}^{\infty} x_{T}(n) e^{j \frac{a}{2 b}(n T)^{2}-j \frac{1}{b} w n} .
\end{aligned}
$$

Here, we use the $\approx$ operator to indicate the discretetime LCT domain.

\subsection{Convolution theorem for the $L C T$}

In [16], the convolution theorem for the LCT has been proposed, which is as follows: Define $y(t)=x(t)^{\mathbf{M}}$ $h(t)=\sqrt{\frac{1}{j 2 \pi b}} e^{-j \frac{a}{2 b} t^{2}}\left[\left(x(t) e^{j \frac{a}{2 b} t^{2}}\right) *\left(h(t) e^{j \frac{a}{2 b} t^{2}}\right)\right]$, where * is the conventional convolution operator for the FT. $\stackrel{\text { M }}{*}$ denotes the convolution for the LCT with the parameter M. $X^{\mathbf{M}}(\mathrm{u}), H^{\mathbf{M}}(\mathrm{u})$, and $Y^{\mathbf{M}}(\mathrm{u})$ are the LCTs of $\mathrm{x}(\mathrm{t})$, $\mathrm{h}(\mathrm{t})$, and $\mathrm{y}(\mathrm{t})$, respectively. Then, $Y^{\mathbf{M}}(u)=X^{\mathbf{M}}(u) \cdot G^{\mathbf{M}}(u)$, where $G^{\mathbf{M}}(u)=H^{\mathbf{M}}(u) e^{-j \frac{d}{2 b} u^{2}}$. Through the convolution theorem for the LCT, we can define a multiplicative filter $G^{\mathbf{M}}(\mathrm{u})$ in the LCT domain, which is a linear time-variant system.

\section{Sampling and interpolation identities associated with the LCT}

In [9], the close analogy between sampling theorems and the multirate filterbanks is exploited, and in [10], the filterbank interpretation of multichannel sampling is given more explicitly by using two identities. The sampling and interpolation identities in [10] are suitable for bandlimited signals in the traditional FT domain. However, these identities are not applicable to signals nonbandlimited in the FT domain, but bandlimited in the LCT domain. In order to solve this problem, we introduce the sampling identity and the interpolation identity for bandlimited signals in the LCT domain in this section.

Theorem 1 Assume that $x(t)$ is a continuous-time signal bandlimited to $\sigma_{M}$ in the LCT domain, $H^{\mathrm{M}}(\mathrm{u})$ is a filter bandlimited to $\sigma_{M}$ in the LCT domain, and T $=N$ $T_{\mathrm{s}}^{\mathrm{M}}$, where $N$ is an integer. Then, the block diagrams illustrated in Figure $1 a, b$ are equivalent, provided that $\tilde{H}^{\mathbf{M}}(w)$ satisfies

$$
\tilde{H}^{\mathrm{M}}(w)=H^{\mathrm{M}}\left(\frac{w}{T_{\mathrm{s}}^{\mathrm{M}}}\right), \quad|w| \leq \pi b .
$$

Proof For simplicity, the parameter matrix $\mathbf{M}$ is omitted in this proof. In Figure 1a, the LCT of $s_{1}(t)$ is

$$
S_{1}(u)=L^{\mathbf{M}}\left[s_{1}\right](u)=X(u) H(u) .
$$

Since $y_{\mathrm{T}}(n)$ is the uniformly sampled version of $s_{1}(t)$ with a period $T$, its DTLCT is

$$
\begin{aligned}
& \tilde{Y}_{T}(w)=\tilde{L}_{T}^{\mathbf{M}}[y](w) \\
& =\frac{1}{T} e^{j \frac{d}{2 b} \frac{w^{2}}{T^{2}}} \sum_{k=-\infty}^{\infty} S_{1}\left(\frac{w}{T}-k \frac{2 \pi}{T} b\right) e^{-j \frac{d}{2 b} \frac{(w-k 2 \pi b)^{2}}{T^{2}}} \\
& =\frac{1}{T} e^{j \frac{d}{2 b} \frac{w^{2}}{T^{2}}} \sum_{k=-\infty}^{\infty} X\left(\frac{w}{T}-k \frac{2 \pi}{T} b\right) H\left(\frac{w}{T}-k \frac{2 \pi}{T} b\right) \\
& e^{-j \frac{d}{2 b} \frac{(w-k 2 \pi b)^{2}}{T^{2}}}
\end{aligned}
$$

where $w=u T$. Note that the bandwidth of $H^{\mathrm{M}}(\mathrm{u})$ in the LCT domain is $\sigma_{\mathbf{M}}$, and $T=N T_{\mathrm{s}}^{\mathbf{M}}=N \pi b / \sigma_{\mathbf{M}}$. Let $k=1+$ $k^{\prime} N$, where $0 \leq l \leq N-1$. Then, (6) becomes

$$
\begin{array}{r}
\tilde{Y}_{T}(w)=\frac{1}{T} e^{j \frac{d w^{2}}{2 b T^{2}}} \sum_{k^{\prime}=-\infty}^{\infty} \sum_{l=0}^{N-1} X\left(\frac{w}{T}-l \frac{2 \pi}{T} b-k^{\prime} \frac{2 \pi}{T_{s}} b\right) \\
H\left(\frac{w}{T}-l \frac{2 \pi}{T} b-k^{\prime} \frac{2 \pi}{T_{s}} b\right) e^{-j \frac{d\left(w-l 2 \pi b-k^{\prime} N 2 \pi b\right)^{2}}{T^{2}}}
\end{array}
$$

In Figure $1 \mathrm{~b}$, the DTLCT of the discrete-time signal $s_{2, T_{\mathrm{s}}^{\mathrm{M}}}(n)$ is

$$
\begin{aligned}
\tilde{S}_{2, T_{\mathrm{s}}^{\mathrm{M}}}\left(w_{\mathrm{s}}\right) & =\tilde{L}_{T_{\mathrm{s}}}^{\mathrm{M}}\left[s_{2}\right]\left(w_{\mathrm{s}}\right) \\
& =\frac{1}{T_{\mathrm{s}}} e^{j \frac{d}{2 b\left(T_{\mathrm{s}}\right)^{2}}} \sum_{k=-\infty}^{\infty} X\left(\frac{w_{\mathrm{s}}}{T_{\mathrm{s}}}-k \frac{2 \pi}{T_{\mathrm{s}}} b\right) e^{-j \frac{d\left(w_{\mathrm{s}}-k \pi n b\right)^{2}}{2\left(T_{\mathrm{s}}\right)^{2}}},
\end{aligned}
$$

where $w_{\mathrm{s}}=u T_{\mathrm{s}}^{\mathbf{M}}$. Thus, we have

$$
\tilde{S}_{\mathrm{H}, T_{\mathrm{s}}^{\mathrm{M}}}\left(w_{\mathrm{s}}\right)=\tilde{S}_{2, T_{\mathrm{s}}^{\mathrm{M}}}\left(w_{\mathrm{s}}\right) \tilde{H}\left(w_{\mathrm{s}}\right), \quad\left|w_{\mathrm{s}}\right| \leq \pi b
$$

The output $y_{T}(n)$ is the result of decimating $s_{\mathrm{H}, T_{\mathrm{s}}^{\mathrm{M}}}(n)$ by a factor $\mathrm{N}$, and thus the DTLCT of $y_{T}(n)$ can be expressed in terms of the spectrum of $s_{\mathrm{H}, T_{\mathrm{s}}^{\mathrm{M}}}(n)$ in the LCT domain:

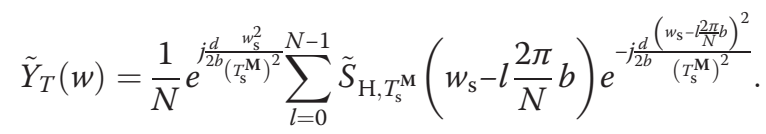


Substituting (8) and (9) into (10), we can obtain

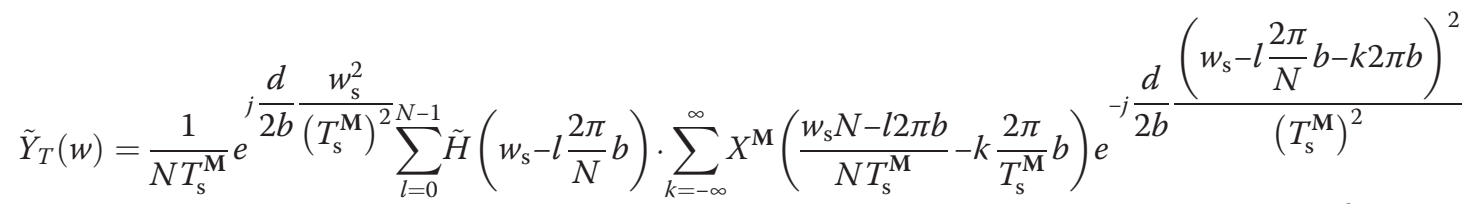

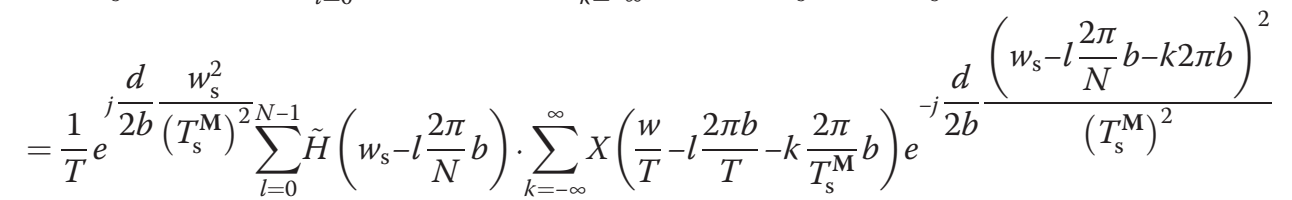

$$
\begin{aligned}
& =\frac{1}{T} e^{j \frac{d}{2 b} \frac{w_{\mathrm{s}}^{2}}{\left(T_{\mathrm{s}}^{\mathbf{M}}\right)^{2}} \sum_{l=0}^{N-1} \sum_{k=-\infty}^{\infty} X\left(\frac{w}{T}-l \frac{2 \pi b}{T}-k \frac{2 \pi}{T_{\mathrm{s}}^{\mathbf{M}}} b\right) e^{-j \frac{d}{2 b} \frac{\left(w_{\mathrm{s}}-l \frac{2 \pi}{N} b-k 2 \pi b\right)^{2}}{\left(T_{\mathrm{s}}^{\mathbf{M}}\right)^{2}}} \tilde{H}\left(w_{\mathrm{s}}-l \frac{2 \pi}{N} b-k \cdot 2 \pi\right) .}
\end{aligned}
$$

Comparing (11) to (7), we observe that the two outputs are equal if

$$
\tilde{H}(w)=H\left(\frac{w}{T_{\mathrm{s}}^{\mathbf{M}}}\right), \quad|w| \leq \pi b .
$$

Theorem 2 Assume that $H(u)$ is a filter bandlimited to $\sigma_{M}$ in the LCT domain, and $H_{\mathrm{LP}}^{\mathrm{M}}(u)$ is a lowpass filter in the LCT domain such that

$$
H_{\mathrm{LP}}^{\mathrm{M}}(u)=\left\{\begin{array}{lc}
T_{\mathrm{s}}^{\mathrm{M}} & |u| \leq \sigma_{\mathrm{M}} \\
0 & \text { otherwise }
\end{array} .\right.
$$

Let $T / T_{s}^{\mathbf{M}}=N$ where $N$ is an integer, then the block diagrams depicted in Figure $2 a, b$ are equivalent, provided that $\tilde{H}^{\mathbf{M}}(w)$ satisfies

$$
\tilde{H}^{\mathbf{M}}(w)=\frac{1}{T_{\mathrm{s}}^{\mathbf{M}}} H^{\mathbf{M}}\left(\frac{w}{T_{\mathrm{s}}^{\mathbf{M}}}\right), \quad|w| \leq \pi b .
$$

Proof For simplicity, the parameter matrix $\mathbf{M}$ is omitted in this proof. In Figure $2 \mathrm{a}$, the input of the filter $H^{\mathrm{M}}(u)$ is

$$
\begin{aligned}
s(t) & =\sum_{n=-\infty}^{\infty} x(n T) \delta(t-n T) \\
& =x(t) \sum_{n=-\infty}^{\infty} \delta(t-n T) .
\end{aligned}
$$

The LCT of $s(t)$ with the parameter $\mathbf{M}$ is

$$
\begin{aligned}
S_{T}(u) & =L_{T}^{\mathbf{M}}[s](u) \\
& =\frac{1}{T} e^{j \frac{d}{2 b} u^{2}} \sum_{k=-\infty}^{\infty} X\left(u-k \frac{2 \pi}{T} b\right) e^{-j \frac{d}{2 b}\left(u-k \frac{2 \pi}{T} b\right)^{2}} .
\end{aligned}
$$

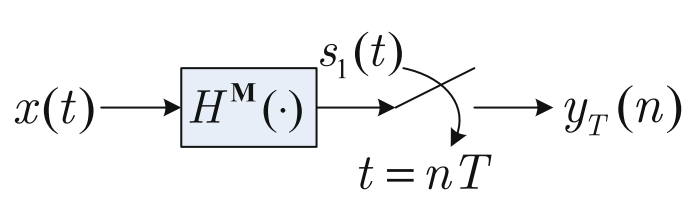

(a)

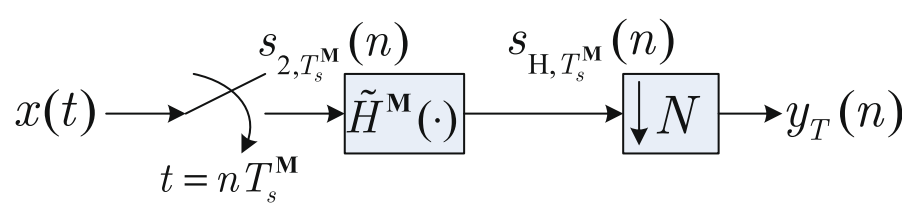

(b)

Figure 1 Sampling identity for bandlimited signals in the LCT domain. (a) Sampling block in continuous-time domain. (b) Sampling block in discrete-time domain. 


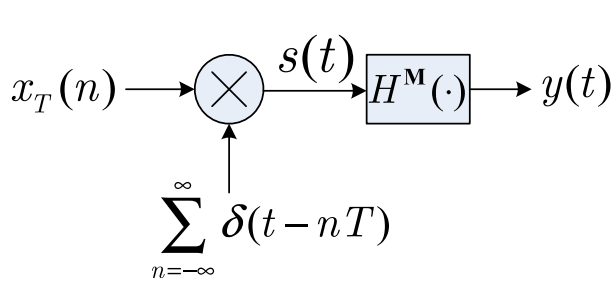

(a)

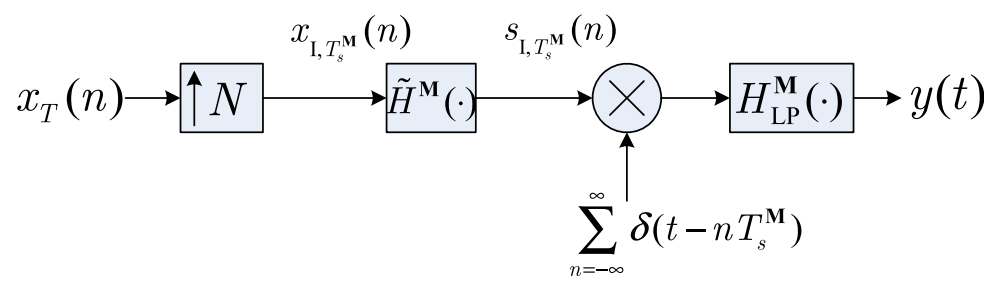

(b)

Figure 2 Interpolation identity for bandlimited signals in the LCT domain. (a) Interpolation block in continuous-time domain.

(b) Interpolation block in discrete-time domain.

Then, we have

$$
\begin{aligned}
Y(u) & =S(u) H(u) \\
& =\frac{1}{T} H(u) e^{j \frac{d}{2 b} u^{2}} \sum_{k=-\infty}^{\infty} X\left(u-k \frac{2 \pi}{T} b\right) e^{-j \frac{d}{2 b}\left(u-k \frac{2 \pi}{T} b\right)^{2}}, \quad|u| \leq \sigma_{\mathbf{M}} .
\end{aligned}
$$

In Figure $2 \mathrm{~b}$, the DTLCT of $x_{T}(n)$ is

$$
\begin{aligned}
\tilde{X}_{T}(w) & =\tilde{L}_{T}^{\mathbf{M}}[x](w) \\
& =\frac{1}{T} e^{j \frac{d w^{2}}{2 b T^{2}}} \sum_{k=-\infty}^{\infty} X\left(\frac{w}{T}-k \frac{2 \pi}{T} b\right) e^{-j \frac{d(w-k 2 \pi b)^{2}}{T^{2}}},
\end{aligned}
$$

where $w=u T$. According to the relationship between the spectrum in the LCT domain and the expansion operation, we have

$$
\begin{aligned}
\tilde{X}_{\mathrm{I}, T_{\mathrm{s}}^{\mathrm{M}}}\left(w_{\mathrm{s}}\right) & =\tilde{X}_{T}\left(w_{\mathrm{s}} N\right) \\
& =\frac{1}{T} e^{j \frac{d\left(w_{\mathrm{s}} N\right)^{2}}{T^{2}}} \sum_{k=-\infty}^{\infty} X\left(\frac{w}{T_{\mathrm{s}}^{\mathrm{M}}}-k \frac{2 \pi}{T} b\right) e^{-j \frac{d\left(w_{\mathrm{s}} N-k 2 \pi b\right)^{2}}{T^{2}}},
\end{aligned}
$$

where $w_{\mathrm{s}}=u T_{\mathrm{s}}^{\mathrm{M}}$. Thus,

$$
\begin{aligned}
\tilde{S}_{\mathrm{I}, T_{\mathrm{s}}^{\mathrm{M}}}\left(w_{\mathrm{s}}\right) & =\tilde{X}_{\mathrm{I}, T_{\mathrm{s}}}\left(w_{\mathrm{s}}\right) \tilde{H}\left(w_{\mathrm{s}}\right) \\
& =\frac{1}{T} \tilde{H}\left(w_{\mathrm{s}}\right) e^{\frac{d d\left(w_{\mathrm{s}} N\right)^{2}}{T^{2}}} \sum_{k=-\infty}^{\infty} X\left(\frac{w}{T_{\mathrm{s}}^{\mathrm{M}}}-k \frac{2 \pi}{T} b\right) e^{-\frac{d\left(w_{\mathrm{s}} N-k 2 \pi b\right)^{2}}{T^{2}}} .
\end{aligned}
$$

Note that $\mathrm{y}(\mathrm{t})$ is a lowpass version of a uniform sampled signal $s_{\mathrm{I}}(t) \sum_{n=-\infty}^{\infty} \delta\left(t-n T_{\mathrm{s}}^{\mathrm{M}}\right)$ with the lowpass filter $H_{\mathrm{LP}}^{\mathrm{M}}(u)$ satisfying (12). Then, we can obtain

$$
\begin{aligned}
Y(u)= & T_{\mathrm{s}}^{\mathbf{M}} \tilde{S}_{\mathrm{I}, T_{\mathrm{s}}}\left(T_{\mathrm{s}}^{\mathbf{M}} u\right) \\
= & \frac{T_{\mathrm{s}}^{\mathbf{M}}}{T} \tilde{H}\left(T_{\mathrm{s}}^{\mathbf{M}} u\right) e^{j \frac{d}{2 b} u^{2}} \sum_{k=-\infty}^{\infty} X\left(u-k \frac{2 \pi}{T} b\right) e^{-j \frac{d(u T-k 2 \pi b)^{2}}{T^{2}}}, \\
& |u| \leq \sigma_{\mathbf{M}} .
\end{aligned}
$$

Comparing (20) with (16), we observe that the two outputs are equal if

$$
H(u)=T_{\mathrm{s}}^{\mathbf{M}} \tilde{H}\left(T_{\mathrm{s}}^{\mathbf{M}} u\right), \quad|u| \leq \sigma_{\mathbf{M}}=\frac{\pi b}{T_{\mathrm{s}}^{\mathbf{M}}}
$$

or equivalently $\tilde{H}(w)=\frac{1}{T_{\mathrm{s}}^{\mathrm{M}}} H\left(\frac{w}{T_{\mathrm{s}}^{\mathrm{M}}}\right), \quad|w| \leq \pi b$.

In Figures 1a and 2a, the whole processing is performed in the continuous-time domain, whereas in Figures $1 \mathrm{~b}$ and $2 \mathrm{~b}$, most of the processing is performed in the discretetime domain. The sampling identity and the interpolation identity establish an equivalence between a continuoustime filtering and a discrete-time filtering involving sampling rate conversion in the LCT domain.

\section{Continuous and discrete LCT filterbanks}

In subband coding for speech and image signals, it is possible to compress the signal by the filterbank for

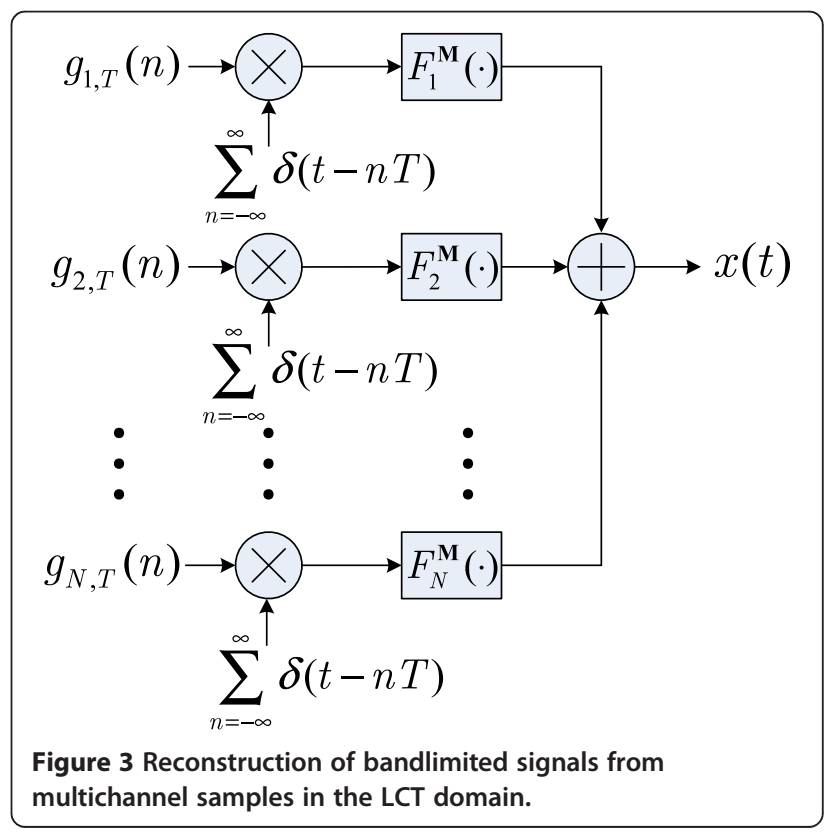




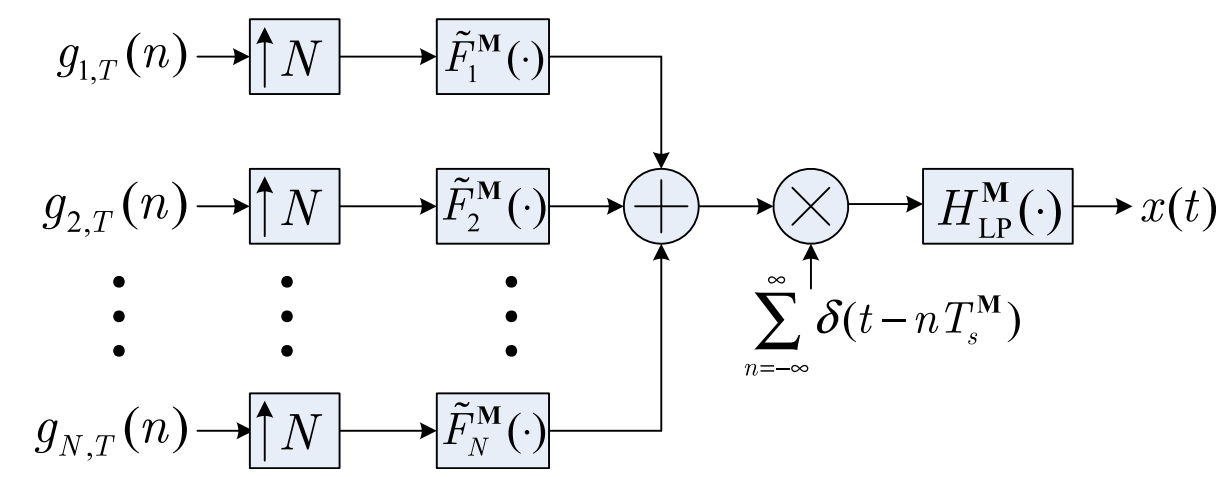

Figure 4 Reconstruction from multichannel samples using the discrete-time LCT filterbank.

bandlimited signals in the FT domain. However, we may encounter signals that are not bandlimited in the FT domain but bandlimited in a LCT domain. In this case, we need to use the filterbank in the LCT domain to perform the subband coding.

In [26] and [30], the Papoulis' multichannel sampling theorem has been extended for bandlimited signals in the FRFT domain and the LCT domain, respectively. To illustrate the utility of the derived identities, we apply them to obtain an efficient discrete-time reconstruction method for the multichannel sampling pattern in the LCT domain in this section. By employing the sampling and interpolation identities for bandlimited signals in the LCT domain, a continuous-time LCT filterbank, which corresponds to a certain multichannel sampling scheme, can be converted to an equivalent discrete-time LCT filterbank and vice versa.

Multichannel sampling theorem in the LCT domain states that a bandlimited signal in the LCT domain can be reconstructed from the samples of the outputs of multiple LCT filters [26]. Let $x(t)$ be a $\sigma_{\mathbf{M}}$ bandlimited signal in the LCT domain with the parameter $\mathbf{M}$, and $H_{k}^{\mathbf{M}}(u), k=1, \ldots, N$ be $N$ filters in the LCT domain. Then, the original input signal $x(t)$ can be reconstructed from the samples of the $N$ outputs $g_{k}(t)$ of the LCT filters $H_{k}^{\mathbf{M}}(u)$. The reconstruction formula is

$$
x(t)=e^{-j \frac{a}{2 b} t^{2}} \sum_{k=1}^{N} \sum_{n=-\infty}^{\infty} g_{k, T}(n) e^{j \frac{a}{2 b}(n T)^{2}} y_{k}(t-n T),
$$

where $T=N T_{\mathrm{s}}^{\mathbf{M}}=N \pi b / \sigma_{\mathbf{M}}, g_{k, T}(\mathrm{n})$ is the uniform samples of $g_{k}(t)$ with a sampling period T, i.e., $g_{k, T}(n)=g_{k}(\mathrm{nT})$, and $y_{k}(t)$ is the reconstruction function. Expressing (22) as a convolution form in the LCT domain, we have

$$
x(t)=\sum_{k=1}^{N}\left[g_{k}(t) \sum_{n=-\infty}^{\infty} \delta(t-n T)\right] \stackrel{\mathbf{M}}{*} z_{k}(t),
$$

where $z_{k}(t)=y_{k}(t) e^{-j \frac{a}{2 b} t^{2}}$.

Let $F_{k}^{\mathbf{M}}(u)=e^{-j \frac{d}{2 b} u^{2}} L^{\mathbf{M}}\left[z_{k}\right](u)$. Then, the reconstruction formula (23) can be achieved via a continuous-time synthesis filterbank that is shown in Figure 3.

Here, the filtering function $F_{k}^{\mathbf{M}}(u)$ denotes the convolution in the LCT domain with the parameter $\mathbf{M}$ rather than the conventional Fourier convolution. It should be pointed that the filters $H_{k}^{\mathbf{M}}(u)$ are general, but not

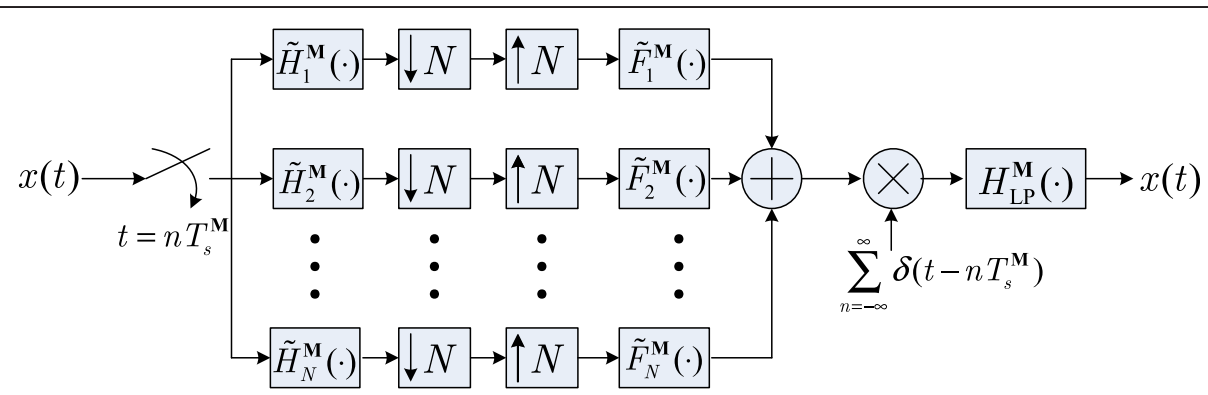

Figure 5 Discrete-time LCT filterbank implementation of sampling and reconstruction processes for bandlimited signals in the LCT domain. 
entirely arbitrary. They must meet the condition that the reconstruction function $y_{k}(t)$ is a solution of the system [26]. Similar condition holds for the filters $F_{k}^{\mathrm{M}}(u)$.

Applying the interpolation identity to each branch in Figure 3 and moving the term $\sum_{n=-\infty}^{\infty} \delta\left(t-n T_{\mathrm{s}}\right)$ and lowpass filter $H_{\mathrm{LP}}^{\mathrm{M}}(u)$ outside the summation for each branch, we can obtain the equivalent implementation shown in Figure 4.

The discrete-time filters $\tilde{F}_{k}^{\mathbf{M}}(w)$ with the expansion operations construct a discrete-time synthesis filterbank or a multirate filterbank in the LCT domain, which can be performed very efficiently (e.g., utilizing the polyphase structure [28]). Since the Papoulis' multichannel sampling theorem is a unified framework for linear filtering operations prior to sampling and the LCTs are a family of integral transforms, reconstruction processes for bandlimited signals in the corresponding transform domain (special cases of the LCT domain) from certain multichannel samples can be efficiently implemented in a similar way.

As the case in the Fourier domain, multichannel sampling for bandlimited signals in the LCT domain can be understood by relating the sampling and reconstruction schemes to the continuous-time analysis/synthesis filterbank system in the LCT domain. Both the LCT continuous-time and discrete-time filterbanks are related to perfect reconstruction (PR) of the original bandlimited signal in the LCT domain, with one in the continuoustime domain and the other in the discrete-time domain. Utilizing the sampling identity and the interpolation identity associated with the LCT, we can convert a continuoustime LCT filterbank to an equivalent discrete-time LCT filterbank preceded by a Nyquist rate sampling and followed by an impulse train multiplication and a lowpass filter in the LCT domain, and vice versa. For example, the discrete-time LCT filterbank implementation of multichannel sampling and reconstruction for bandlimited signals in the LCT domain is shown in Figure 5. This relationship also helps us derive new sampling schemes. We can obtain new sampling and reconstruction processes from a PR LCT multirate filterbank by converting it to a continuoustime LCT filterbank.

\section{Conclusions}

This paper investigates the relationship between the multichannel sampling theorem for bandlimited signals in the LCT domain and the LCT multirate filterbanks. Two identities for bandlimited signals in the LCT domain are firstly presented and then applied to obtain the discrete-time implementation for multichannel sampling based on the LCT. Through the use of the sampling and the interpolation identities associated with the LCT, we establish a relationship between the continuous-time LCT filterbank and the discrete-time LCT filterbank. New sampling strategies based on the LCT can be obtained using these identities. Since the discrete-time filterbank can be implemented efficiently (e.g., using the polyphase structure), the sampling and reconstruction processes can be realized efficiently.

\section{Competing interests}

The authors declare that they have no competing interests.

\section{Acknowledgments}

This work was supported in part by the National Science Foundation of China for Distinguished Young Scholars under grant 60625104, by the National Natural Science Foundation of China under grant 61201354, by the Basic Science Foundation of Beijing Institute of Technology under grant 20120542005, and by the National Key Basic Research Program Founded by MOST under grants 2009CB724003 and 2010CB731902.

Received: 23 December 2012 Accepted: 25 April 2013

Published: 11 May 2013

\section{References}

1. PP Vaidyanathan, Multirate digital filters, filter banks, polyphase networks, and applications: a tutorial. Proc. IEEE. 78(1), 56-93 (1990)

2. S-M Phoong, PP Vaidyanathan, Time-varying filters and filter banks: some basic principles. IEEE Trans. Signal Process. 44(12), 2971-2987 (1996)

3. JW Woods, SD Neil, Subband coding images. IEEE Trans. Acoust. Speech Signal Process. 34(5), 1278-1288 (1986)

4. M Vetterli, Multi-dimensional sub-band coding: some theory and algorithm. Signal Process. 6(2), 97-112 (1984)

5. H Gharavi, A Tabatabai, Sub-band coding of monochrome and color images. IEEE Trans. Circuits Syst. 35(2), 207-214 (1988)

6. M Unser, Sampling-50 years after Shannon. Proc. IEEE 88(4), 569-587 (2000)

7. A Papoulis, Generalized sampling expansion. IEEE Trans. Circuits Syst. cas- 24(11), 652-654 (1977)

8. JL Brown, Multichannel sampling of lowpass signals. IEEE Trans. Circuits Syst. cas- 28(2), 101-106 (1981)

9. PP Vaidyanathan, VC Liu, Classical sampling theorems in the context of multirate and polyphase digital filter bank structures. IEEE T. Acoust. Speech. 36(9), 1480-1495 (1988)

10. YC Eldar, AV Oppenheim, Filterbank reconstruction of bandlimited signals from nonuniformly and generalized samples. IEEE. T. Signal. Process. 48(10), 2864-2875 (2000)

11. HM Ozaktas, Z Zalevsky, MA Kutay, The Fractional Fourier Transform with Applications in Optics and Signal Processing (Wiley, New York, 2000)

12. A Stern, Why is the linear canonical transform so little known? Proc. AIP 860, 225-234 (2006)

13. DFV James, GS Agarwal, The generalized Fresnel transform and its applications to optics. Opt. Commun. 126(5), 207-212 (1996)

14. A Koc, HM Ozaktas, C Candan, MA Kutay, Digital computation of linear canonical transforms. IEEE T. Signal. Process. 56(6), 2383-2394 (2008)

15. SC Pei, JJ Ding, Eigenfunctions of linear canonical transform. IEEE T. Signal. Process. 50(1), 11-26 (2002)

16. B Deng, $\mathrm{R}$ Tao, $\mathrm{Y}$ Wang, Convolution theorems for the linear canonical transform and their applications. Science in China Ser. F 49(5), 592-603 (2006)

17. L Onural, A Gotchev, HM Ozaktas, E Stoykova, A survey of signal processing problems and tools in holographic three-dimensional television. IEEE Trans. Circuit Syst. Video Technol. 17(11), 1631-1646 (2007)

18. A Stern, Uncertainty principles in linear canonical transform domain and some of their implications in optics. J. Opt. Soc. Am. A 25(3), 647-652 (2008)

19. KK Sharma, SD Joshi, Uncertainty principle for real signals in the linear canonical transform domains. IEEE. T. Signal. Process. 56(7), 2677-2683 (2008)

20. A Stern, Sampling of linear canonical transformed signals. Signal Process. 86(7), 1421-1425 (2006)

21. BZ Li, R Tao, Y Wang, New sampling formulae related to linear canonical transform. Signal Process. 87(5), 983-990 (2007) 
22. JJ Healy, JT Sheridan, Sampling and discretization of the linear canonical transform. Signal Process. 89(4), 641-648 (2009)

23. B Lacaze, About sampling for band-limited linear canonical transform. Signal Process. 91(4), 1076-1078 (2011)

24. KK Sharma, Vector sampling expansions and linear canonical transform. IEEE. Signal. Proc. Let. 18(10), 583-586 (2011)

25. R Tao, BZ Li, Y Wang, GK Aggrey, On sampling of band-limited signals associated with the linear canonical transform. IEEE. T. Signal. Process. 56(11), 5454-5464 (2008)

26. DY Wei, QW Ran, YM Li, Reconstruction of band-limited signals from multichannel and periodic nonuniform samples in the linear canonical transform domain. Opt. Commun. 284(19), 4307-4315 (2011)

27. J Zhao, R Tao, Y Wang, Sampling rate conversion for linear canonical transform. Signal Process. 88(11), 2825-2832 (2008)

28. S Shinde, Two channel paraunitary filter banks based on linear canonical transform. IEEE. T. Signal. Process. 59(2), 832-836 (2011)

29. JJ Healy, JT Sheridan, Cases where the linear canonical transform of a signal has compact support or is band-limited. Opt. Lett. 33(3), 228-230 (2008)

30. KK Sharma, SD Joshi, Papoulis-like generalized sampling expansions in fractional Fourier domains and its application to superresolution. Opt. Commun. 278(1), 52-59 (2007)

doi:10.1186/1687-6180-2013-102

Cite this article as: Zhang et al:: Relationship between sampling and multirate filterbanks in the linear canonical transform domain. EURASIP Journal on Advances in Signal Processing 2013 2013:102.

Submit your manuscript to a SpringerOpen ${ }^{\circ}$ journal and benefit from:

- Convenient online submission

- Rigorous peer review

- Immediate publication on acceptance

- Open access: articles freely available online

- High visibility within the field

- Retaining the copyright to your article

Submit your next manuscript at springeropen.com 